\title{
Tezepelumab in Adults and Adolescents with Severe, Uncontrolled Asthma
}

\author{
Andrew Menzies-Gow, M.D., Jonathan Corren, M.D., Arnaud Bourdin, M.D., \\ Geoffrey Chupp, M.D., Elliot Israel, M.D., Michael E. Wechsler, M.D., \\ Christopher E. Brightling, F.Med.Sci., Janet M. Griffiths, Ph.D., \\ Åsa Hellqvist, M.Sc., Karin Bowen, M.Sc., Primal Kaur, M.D., \\ Gun Almqvist, M.Sc., Sandhia Ponnarambil, M.D., and Gene Colice, M.D.
}

\section{ABSTRACT}

From Royal Brompton Hospital, London (A.M.-G.), Leicester National Institute for Health Research Biomedical Research Centre, University of Leicester, Leicester (C.E.B.), and Late-stage Development, Respiratory and Immunology, BioPharmaceuticals R\&D, AstraZeneca, Cambridge (S.P.) - all in the United Kingdom; the David Geffen School of Medicine, University of California, Los Angeles, Los Angeles (J.C.), and Global Development, Amgen, Thousand Oaks (P.K.) - both in California; Physiologie et Médecine Expérimentale du Cœur et des Muscles, Université de Montpellier, Centre National de la Recherche Scientifique, INSERM, Centre Hospitalier Universitaire de Montpellier, Montpellier, France (A.B.); the Division of Pulmonary, Critical Care, and Sleep Medicine, Department of Medicine, Yale School of Medicine, New Haven, CT (G. Chupp); the Division of Pulmonary and Critical Care Medicine and Allergy and Immunology, Brigham and Women's Hospital, Harvard Medical School, Boston (E.I.); National Jewish Health, Denver (M.E.W.); Translational Science and Experimental Medicine, Research and Early Development, Respiratory and Immunology (J.M.G.), and Biometrics (K.B.), Late-stage Development, Respiratory and Immunology (G. Colice), BioPharmaceuticals R\&D, AstraZeneca, Gaithersburg, MD; and Biometrics (Å.H.), Late-stage Development, Respiratory and Immunology (G.A.), BioPharmaceuticals R\&D, AstraZeneca, Gothenburg, Sweden. Address reprint requests to Dr. Menzies-Gow at Royal Brompton Hospital, Sydney St., London SW3 6NP, United Kingdom, or at a.menzies-gow@ rbht.nhs.uk.

\section{BACKGROUND}

Tezepelumab is a human monoclonal antibody that blocks thymic stromal lymphopoietin, an epithelial-cell-derived cytokine implicated in the pathogenesis of asthma. The efficacy and safety of tezepelumab in patients with severe, uncontrolled asthma require further assessment.

\section{METHODS}

We conducted a phase 3, multicenter, randomized, double-blind, placebo-controlled trial. Patients (12 to 80 years of age) were randomly assigned to receive tezepelumab (210 mg) or placebo subcutaneously every 4 weeks for 52 weeks. The primary end point was the annualized rate of asthma exacerbations over a period of 52 weeks. This end point was also assessed in patients with baseline blood eosinophil counts of less than 300 cells per microliter. Secondary end points included the forced expiratory volume in 1 second $\left(\mathrm{FEV}_{1}\right)$ and scores on the Asthma Control Questionnaire-6 (ACQ-6; range, 0 [no impairment] to 6 [maximum impairment]), Asthma Quality of Life Questionnaire (AQLQ; range, 1 [maximum impairment] to 7 [no impairment]), and Asthma Symptom Diary (ASD; range, 0 [no symptoms] to 4 [worst possible symptoms]).

RESULTS

Overall, 1061 patients underwent randomization (529 were assigned to receive tezepelumab and 532 to receive placebo). The annualized rate of asthma exacerbations was 0.93 (95\% confidence interval [CI], 0.80 to 1.07) with tezepelumab and 2.10 (95\% CI, 1.84 to 2.39 ) with placebo (rate ratio, $0.44 ; 95 \% \mathrm{CI}, 0.37$ to $0.53 ; \mathrm{P}<0.001$ ). In patients with a blood eosinophil count of less than 300 cells per microliter, the annualized rate was 1.02 (95\% CI, 0.84 to 1.23) with tezepelumab and 1.73 (95\% CI, 1.46 to 2.05 ) with placebo (rate ratio, 0.59 ; $95 \%$ CI, 0.46 to 0.75 ; $\mathrm{P}<0.001$ ). At week 52 , improvements were greater with tezepelumab than with placebo with respect to the prebronchodilator $\mathrm{FEV}_{1}$ ( 0.23 vs. 0.09 liters; difference, 0.13 liters; $95 \% \mathrm{CI}, 0.08$ to $0.18 ; \mathrm{P}<0.001)$ and scores on the ACQ-6 ( -1.55 vs. -1.22 ; difference, -0.33 ; $95 \%$ $\mathrm{CI},-0.46$ to -0.20 ; P<0.001), AQLQ (1.49 vs. 1.15 ; difference, $0.34 ; 95 \% \mathrm{CI}, 0.20$ to 0.47 ; $\mathrm{P}<0.001)$, and ASD ( -0.71 vs. -0.59 ; difference, -0.12 ; $95 \% \mathrm{CI},-0.19$ to -0.04 ; $\mathrm{P}=0.002$ ). The frequencies and types of adverse events did not differ meaningfully between the two groups.

\section{CONCLUSIONS}

Patients with severe, uncontrolled asthma who received tezepelumab had fewer exacerbations and better lung function, asthma control, and health-related quality of life than those who received placebo. (Funded by AstraZeneca and Amgen; NAVIGATOR ClinicalTrials.gov number, NCT03347279.) 
PPROXIMATELY $10 \%$ OF PATIENTS WITH asthma have severe disease and have symptoms and exacerbations despite treatment with maximal standard-of-care controller therapy. ${ }^{1}$ Severe, uncontrolled asthma contributes disproportionately to the overall burden and cost of asthma. ${ }^{2}$ Monoclonal antibodies that target IgE or type 2 (T2) cytokines (interleukin-4, -5, and -13) and their receptors improve disease control for many patients with severe asthma and are included in management guidelines. ${ }^{1}$ However, current biologic agents are unsuitable for many patients with severe asthma, particularly those with nonallergic or noneosinophilic phenotypes. ${ }^{3,4}$ Thus, there remains an unmet need for new therapies that are effective in a broader population of patients.

Thymic stromal lymphopoietin (TSLP) is an epithelial-cell-derived cytokine implicated in multiple downstream processes involved in asthma pathophysiology. ${ }^{5-7}$ In patients with asthma, TSLP levels are correlated with airway obstruction, disease severity, and glucocorticoid resistance. ${ }^{8,9}$ In addition to driving $\mathrm{T} 2$ inflammation of the airway, ${ }^{5,6,10}$ TSLP has been shown to mediate interactions between airway structural cells and immune cells, which are not exclusively driven by T2 inflammation. ${ }^{8,11-14}$

Tezepelumab is a human monoclonal antibody (IgG $2 \lambda$ ) that binds specifically to TSLP, blocking it from interacting with its heterodimeric receptor. ${ }^{15}$ In the phase $2 \mathrm{~b}$ PATHWAY trial, the annualized rate of asthma exacerbations was up to $71 \%$ lower with tezepelumab than with placebo among patients with severe, uncontrolled asthma. Exacerbations were reduced irrespective of baseline levels of inflammatory biomarkers (including fraction of exhaled nitric oxide [FENO], blood eosinophils, and IgE) and allergic status. ${ }^{16-19}$ We conducted the NAVIGATOR trial to further investigate the earlier findings in patients with severe, uncontrolled asthma.

\section{METHODS}

\section{PATIENTS}

Patients were 12 to 80 years of age with physiciandiagnosed asthma, who had received mediumor high-dose inhaled glucocorticoids (daily dose of $\geq 500 \mu \mathrm{g}$ of fluticasone propionate or equivalent) for at least 12 months before screening and at least one additional controller medication, with or without oral glucocorticoids, for at least
3 months before the date of informed consent. Physician-diagnosed asthma must have been documented for at least 12 months before screening. The morning prebronchodilator forced expiratory volume in 1 second $\left(\mathrm{FEV}_{1}\right)$ must have been less than $80 \%$ of the predicted normal value $(<90 \%$ for patients 12 to 17 years of age) during the runin period. Postbronchodilator (albuterol) $\mathrm{FEV}_{1}$ reversibility of at least $12 \%$ and at least $200 \mathrm{ml}$ must have been documented during the 12 months before screening or during the run-in period. Patients must have had at least two asthma exacerbations (defined for trial eligibility and end-point measures as a worsening of asthma symptoms that led to hospitalization, an emergency department visit that resulted in the use of systemic glucocorticoids for $\geq 3$ consecutive days, or the use of systemic glucocorticoids for $\geq 3$ consecutive days) in the 12 months before the date of informed consent. Patients who had received marketed or noninvestigational biologic treatments were permitted to enter the trial if the last dose was taken more than 4 months, or more than five half-lives, before screening (whichever was longer).

\section{TRIAL DESIGN}

This was a phase 3, multicenter, double-blind, randomized, placebo-controlled trial conducted from November 23, 2017, to September 8, 2020, at 297 sites in 18 countries. (A list of trial sites is provided in Table S11 in the Supplementary Appendix, available with the full text of this article at NEJM.org.) Full details of the design have been published previously. ${ }^{20}$ After a 5-to-6-week screening and run-in period (to confirm eligibility), patients were randomly assigned in a 1:1 ratio to receive tezepelumab at a dose of $210 \mathrm{mg}$ or placebo (administered with a single-use vial and syringe) subcutaneously every 4 weeks for 52 weeks. Patients were stratified according to geographic region (Asia-Pacific, central and eastern Europe, western Europe and Australia, North America, South America, or the rest of the world) and age (adults or adolescents). The tezepelumab dose was selected on the basis of pharmacokinetic modeling and exposure-response analyses with the use of data from previous clinical studies. ${ }^{21}$ At week 52, patients entered a 12-week post-treatment follow-up period or the long-term extension study (DESTINATION; ClinicalTrials.gov number, NCT03706079).

The trial population was monitored to ensure distribution of patients across three characteris- 
tics: approximately $20 \%$ of patients were to have received stable medium-dose inhaled glucocorticoids in the previous 3 months, approximately $40 \%$ were to have had at least three exacerbations in the previous 12 months, and approximately $50 \%$ were to have a blood eosinophil count of at least 300 cells per microliter. (The blood eosinophil count was assessed centrally, as described in the Supplementary Appendix.) During the trial, all the patients continued to receive their previously prescribed inhaled glucocorticoids plus additional controller medications, with or without oral glucocorticoids, without change. Patients were permitted to use short-acting $\beta_{2}$-agonists for symptom relief as needed.

\section{TRIAL OVERSIGHT}

The trial design was approved by the appropriate national and institutional regulatory authorities and ethics committees, and all the patients or their guardians provided written informed consent. The trial protocol, including the statistical analysis plan, is available at NEJM.org.

An independent data and safety monitoring board reviewed unblinded data every 6 months (and as required) to monitor safety aspects of the trial. AstraZeneca coordinated data management and performed the statistical analysis in collaboration with the authors, all of whom had access to the final trial data. A contract research organization (IQVIA) was involved in data management, coordinated by AstraZeneca. All the authors contributed to designing the trial, interpreting the data, drafting the manuscript (with support from a medical writer funded by the sponsors), and providing critical feedback and final approval for submission. All the authors vouch for the completeness and accuracy of the data and for adherence of the trial to the protocol.

\section{END POINTS}

The primary end point was the annualized rate of asthma exacerbations (events per patient-year) over the 52-week treatment period in the overall population. This end point was also assessed in patients with a baseline blood eosinophil count of less than 300 cells per microliter. Key secondary end points were the change from baseline in the prebronchodilator $\mathrm{FEV}_{1}$ (minimum clinically important difference [MCID], 0.1 liters), ${ }^{22}$ the Asthma Control Questionnaire-6 (ACQ-6) score (range, 0 [no impairment] to 6 [maximum impairment]; MCID, 0.5 points), ${ }^{23}$ the Asthma Qual- ity of Life Questionnaire (standardized) for patients 12 years of age or older (AQLQ[S]+12) overall score (range, 1 [maximum impairment] to 7 [no impairment]; MCID, 0.5 points), ${ }^{24}$ and the weekly mean Asthma Symptom Diary (ASD) score (range, 0 [no symptoms] to 4 [worst possible symptoms]; MCID, 0.5 points). ${ }^{25-27}$ Details of additional secondary end points, including exacerbations associated with hospitalization or an emergency department visit and the change from baseline to week 52 in biomarkers of T2 inflammation, are provided in the Supplementary Appendix.

As part of prespecified analyses, the annualized rate of asthma exacerbations over a period of 52 weeks was assessed in patients grouped according to baseline blood eosinophil count, FenO level, serum-specific IgE status (positive or negative for perennial allergen sensitivity), age, geographic region, dose of inhaled glucocorticoids, use or nonuse of oral glucocorticoids, and age at asthma onset (post hoc). Key secondary end points were also assessed in patients grouped according to baseline blood eosinophil count (see the Supplementary Appendix). Adverse events and serious adverse events that occurred during the trial (from the first receipt of tezepelumab or placebo to trial completion or withdrawal date) were recorded.

\section{STATISTICAL ANALYSIS}

To control the type I error for the primary and key secondary end points, a hierarchical test sequence was used (see the Supplementary Appendix). The primary end point was first tested in the overall population and then in the subgroup of patients with baseline blood eosinophil counts of less than 300 cells per microliter, followed by the key secondary end points.

It was estimated that approximately 530 patients per trial group would confer greater than 99\% power to detect a between-group difference in the annualized rate of asthma exacerbations of $50 \%$ at a two-sided significance level of $1 \%$ in the overall population (primary end point), under the assumption of a rate with placebo of 0.9 per year and a shape parameter of 2.4 (overdispersion), and would confer at least $90 \%$ power at a two-sided significance level of $5 \%$ for the key secondary end points. For the annualized rate of asthma exacerbations among patients with a blood eosinophil count of less than 300 cells per microliter, it was assumed that the rate with placebo would be 0.6 and that half the overall patient population would be in this group (i.e., 265 
patients per trial group), giving $94 \%$ power to detect a between-group difference of $50 \%$ at a two-sided significance level of 5\%.

The annualized rate of asthma exacerbations and the rate of exacerbations associated with hospitalization or an emergency department visit over a period of 52 weeks were analyzed with the use of a negative binomial regression model, with the total number of asthma exacerbations in a patient over the 52-week trial period used as a response variable. Trial-group assignment, geographic region, age, and history of exacerbations were included as covariates. The logarithm of the time at risk for an exacerbation during the trial was used as an offset variable.

The effect of tezepelumab or placebo on the annualized rate of asthma exacerbations over a period of 52 weeks across the continuum of baseline biomarker levels was visualized with the use of locally weighted regression and smoothing scatterplots ${ }^{28}$ for the two trial groups. Missing data were assumed to be missing at random and were modeled on the basis of what was observed during the trial with the use of direct maximum likelihood approaches (see the Supplementary Appendix). Full statistical methods are provided in the statistical analysis plan. Changes from baseline to week 52 for key secondary end points in the two trial groups were compared with the use of a repeated-measures model, after multiple imputation to complete the follow-up with the assumption that data were missing at random. All observations were included in the analyses, including those after discontinuation of tezepelumab or placebo.

\section{RESULTS}

\section{PATIENTS}

Overall, 2420 patients were screened and 1061 underwent randomization: 529 were assigned to receive tezepelumab and 532 to receive placebo. Of these patients, 528 and 531 received tezepelumab and placebo, respectively, and were assessed for efficacy and safety end points (overall population) (Fig. S1). The demographic and clinical characteristics of the patients at baseline were balanced between the trial groups (Table 1 and Table S1).

\section{PRIMARY END POINT}

In the overall population, tezepelumab treatment resulted in an annualized rate of asthma exacerbations of 0.93 ( $95 \%$ confidence interval
[CI], 0.80 to 1.07), as compared with 2.10 (95\% CI, 1.84 to 2.39 ) in the placebo group (rate ratio, $0.44 ; 95 \% \mathrm{CI}, 0.37$ to 0.53 ; $\mathrm{P}<0.001$ ). In patients with a blood eosinophil count of less than 300 cells per microliter, the annualized rate of asthma exacerbations was 1.02 (95\% CI, 0.84 to 1.23) in the tezepelumab group and 1.73 (95\% CI, 1.46 to 2.05 ) in the placebo group (rate ratio, $0.59 ; 95 \%$ $\mathrm{CI}, 0.46$ to 0.75 ; $\mathrm{P}<0.001$ ) (Fig. 1 and Fig. S2). The asthma exacerbations that were used for the primary analysis are summarized in Table S2.

\section{SECONDARY END POINTS}

At week 52, the change from baseline in the prebronchodilator $\mathrm{FEV}_{1}$ was 0.23 liters in the tezepelumab group and 0.09 liters in the placebo group (difference, 0.13 liters; 95\% CI, 0.08 to $0.18 ; \mathrm{P}<0.001$ ) (Table 2). The treatment effect was observed as early as week 2 (the first time point assessed) and was sustained throughout the treatment period (Fig. 2). For the tezepelumab and placebo groups, the change from baseline in the ACQ-6 score was -1.55 and -1.22 , respectively (difference, $-0.33 ; 95 \% \mathrm{CI},-0.46$ to -0.20 ; $\mathrm{P}<0.001)$; in the AQLQ(S)+12 score, the change from baseline was 1.49 and 1.15 , respectively (difference, $0.34 ; 95 \% \mathrm{CI}, 0.20$ to 0.47 ; $\mathrm{P}<0.001$ ); and in the ASD score, the change from baseline was -0.71 and -0.59 , respectively (difference, $-0.12 ; 95 \% \mathrm{CI},-0.19$ to $-0.04 ; \mathrm{P}=0.002)(\mathrm{Ta}-$ ble 2). Improvements were observed from the first postbaseline assessment and were sustained throughout the treatment period (Fig. S3). Results for additional secondary and exploratory end points are shown in Table S3. The rate of exacerbations that were associated with hospitalization or an emergency department visit over a period of 52 weeks was 0.06 with tezepelumab and 0.28 with placebo (rate ratio, $0.21 ; 95 \% \mathrm{CI}$, 0.12 to 0.37 ) (Fig. S4). The time to first exacerbation was longer in the tezepelumab group than in the placebo group (Fig. S5).

Reductions from baseline in blood eosinophil counts and Feno levels in patients who received tezepelumab were observed at week 2 and were sustained throughout the treatment period (Table 2 and Figs. S6A and S6B). Serum total IgE levels gradually decreased over a period of 52 weeks in the tezepelumab group (Table 2 and Fig. S6C).

\section{OTHER PRESPECIFIED EXPLORATORY END POINTS}

The cumulative number of exacerbations over a period of 52 weeks was lower in patients who 


\begin{tabular}{|c|c|c|c|}
\hline Characteristic & $\begin{array}{l}\text { Tezepelumab } \\
(\mathrm{N}=528)\end{array}$ & $\begin{array}{l}\text { Placebo } \\
(\mathrm{N}=531)\end{array}$ & $\begin{array}{c}\text { Total } \\
(\mathrm{N}=1059)\end{array}$ \\
\hline Age $-y r$ & $49.9 \pm 16.3$ & $49.0 \pm 15.9$ & $49.5 \pm 16.1$ \\
\hline Male sex - no. (\%) & $193(36.6)$ & $194(36.5)$ & $387(36.5)$ \\
\hline White race - no. $(\%) \dagger$ & $332(62.9)$ & $327(61.6)$ & $659(62.2)$ \\
\hline Body-mass indext & $28.7 \pm 7.1$ & $28.3 \pm 6.9$ & $28.5 \pm 7.0$ \\
\hline \multicolumn{4}{|l|}{ Dose of inhaled glucocorticoids — no. (\%) } \\
\hline Low & 0 & $1(0.2)$ & $1(0.1)$ \\
\hline Medium & $131(24.8)$ & $132(24.9)$ & $263(24.8)$ \\
\hline High & $397(75.2)$ & $398(75.0)$ & $795(75.1)$ \\
\hline \multicolumn{4}{|l|}{ Use of oral glucocorticoids — no. (\%) } \\
\hline Yes & $49(9.3)$ & $51(9.6)$ & $100(9.4)$ \\
\hline No & $479(90.7)$ & $480(90.4)$ & $959(90.6)$ \\
\hline $\begin{array}{l}\text { Prebronchodilator } \mathrm{FEV}_{1} \text { - \% of predicted } \\
\text { normal value }\end{array}$ & $62.8 \pm 18.0$ & $62.7 \pm 18.0$ & $62.7 \pm 18.0$ \\
\hline ACQ-6 score $\$$ & $2.8 \pm 0.8$ & $2.8 \pm 0.8$ & $2.8 \pm 0.8$ \\
\hline \multicolumn{4}{|l|}{ AQLQ(S)+12 overall score } \\
\hline No. of patients evaluated & 527 & 529 & 1056 \\
\hline Mean & $3.9 \pm 1.0$ & $3.9 \pm 1.0$ & $3.9 \pm 1.0$ \\
\hline \multicolumn{4}{|l|}{ FENO level } \\
\hline No. of patients evaluated & 522 & 527 & 1049 \\
\hline Mean - ppb & $41.4 \pm 36.3$ & $46.3 \pm 44.7$ & $43.8 \pm 40.8$ \\
\hline Median (range) - ppb & $31.0(5.0-235.0)$ & $30.0(5.0-265.0)$ & $30.0(5.0-265.0)$ \\
\hline$<25 \mathrm{ppb}-$ no. $(\%)$ & $213(40.8)$ & $220(41.7)$ & $433(41.3)$ \\
\hline$\geq 25 \mathrm{ppb}-$ no. (\%) & $309(59.2)$ & $307(58.3)$ & $616(58.7)$ \\
\hline \multicolumn{4}{|l|}{ Blood eosinophil count } \\
\hline Mean - cells $/ \mu \mid$ & $327 \pm 293$ & $353 \pm 488$ & $340 \pm 403$ \\
\hline Median (range) — cells $/ \mu \mid$ & $250(0-3650)$ & $250(0-8170)$ & $250(0-8170)$ \\
\hline$<300$ cells $/ \mu \mathrm{l}-$ no. (\%) & $309(58.5)$ & $309(58.2)$ & $618(58.4)$ \\
\hline$\geq 300$ cells $/ \mu 1-$ no. (\%) & $219(41.5)$ & $222(41.8)$ & $441(41.6)$ \\
\hline \multicolumn{4}{|l|}{ Serum total IgE - IU/ml } \\
\hline Mean & $515.7 \pm 959.8$ & $614.1 \pm 1159.5$ & $565.0 \pm 1065.2$ \\
\hline Median (range) & $194.9(1.5-12,823.2)$ & $196.7(1.5-9740.9)$ & $195.6(1.5-12,823.2)$ \\
\hline
\end{tabular}

* Plus-minus values are means \pm SD. Patients were randomly assigned to receive tezepelumab (210 mg) or placebo subcutaneously every 4 weeks. Percentages may not total 100 because of rounding. FENo denotes fraction of exhaled nitric oxide, $\mathrm{FEV}_{1}$ forced expiratory volume in 1 second, and $\mathrm{ppb}$ parts per billion.

$\uparrow$ Race was reported by the patients.

The body-mass index is the weight in kilograms divided by the square of the height in meters.

$\int$ Scores on the Asthma Control Questionnaire-6 (ACQ-6) range from 0 (no impairment) to 6 (maximum impairment), with lower scores indicating better disease control. Scores higher than 1.5 indicate inadequately controlled asthma.

Scores lower than 0.75 indicate well-controlled asthma.

I Scores on the Asthma Quality of Life Questionnaire (standardized) for patients 12 years of age or older (AQLQ[S]+12) range from 1 (maximum impairment) to 7 (no impairment), with higher scores indicating better health-related quality of life.

received tezepelumab than in those who received placebo (Fig. S7). Overall, $43.8 \%$ of the patients who received tezepelumab and $60.1 \%$ of those who received placebo had at least one exacerbation (Table S4).
Tezepelumab treatment resulted in annualized rates of asthma exacerbations of $0.79,1.04$, and 0.89 in patients with a baseline blood eosinophil count of at least 300 , less than 150 , and at least 150 cells per microliter, respectively, as compared 


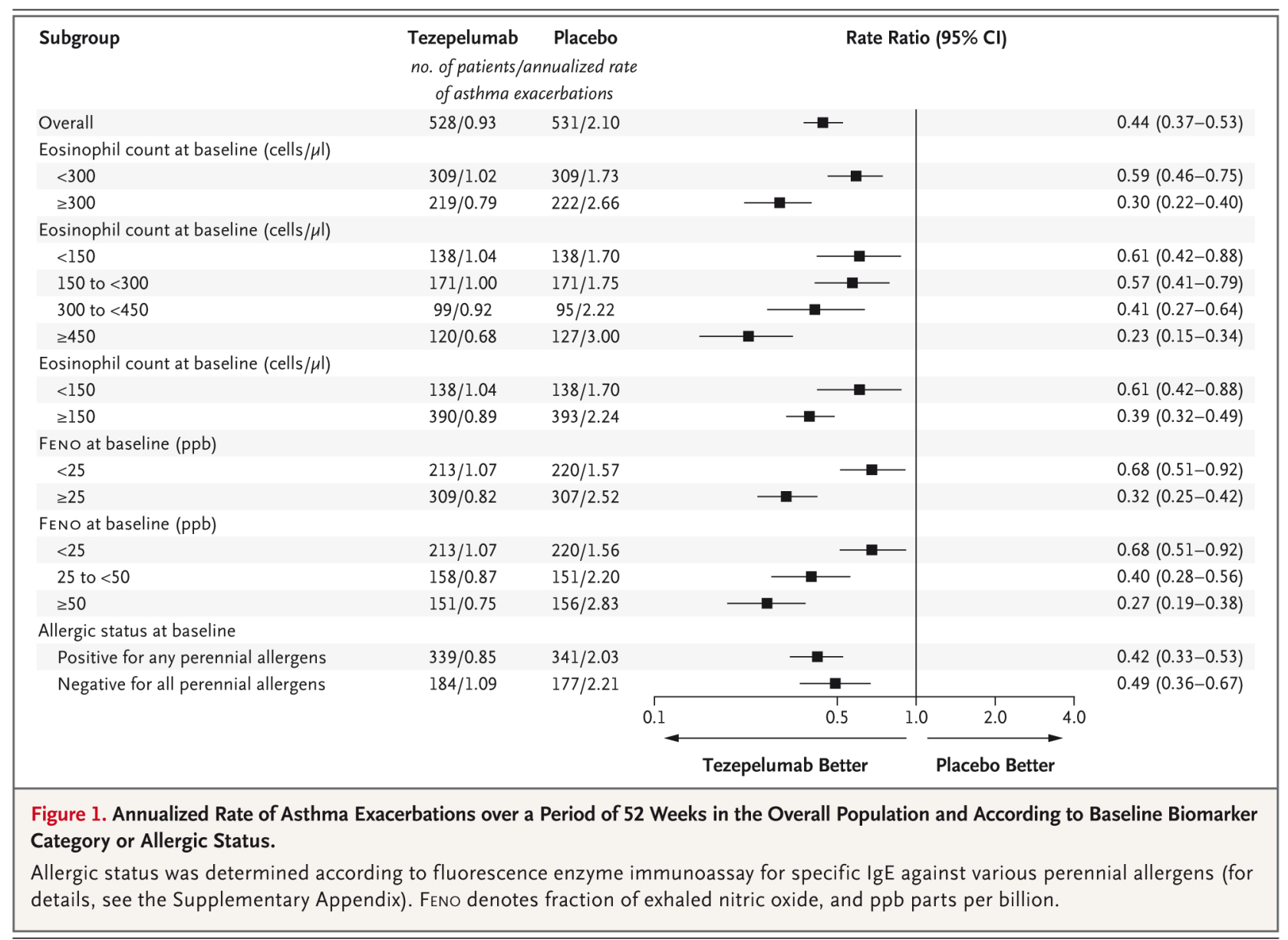

with $2.66,1.70$, and 2.24 in the respective placebo groups (Fig. 1). This corresponded to rate ratios of 0.30 ( $95 \% \mathrm{CI}, 0.22$ to 0.40$), 0.61$ (95\% CI, 0.42 to 0.88 ), and 0.39 ( $95 \% \mathrm{CI}, 0.32$ to 0.49 ), respectively. Annualized rates of asthma exacerbations according to baseline Feno level, perennialallergy status, serum total IgE level, and blood eosinophil count are shown in Figure 1 and Figures S8 and S9. Some variation was observed among geographic regions with respect to treatment effect on the annualized rate of asthma exacerbations over a period of 52 weeks (Fig. S10). Additional subgroups that were assessed are shown in Figure S10. When $\mathrm{FEV}_{1}$ and scores on the ACQ- $6, \mathrm{AQLQ}(\mathrm{S})+12$, and $\mathrm{ASD}$ were analyzed according to baseline blood eosinophil count, the greatest improvements in patients who received tezepelumab were observed in those with a count of at least 300 cells per microliter (Table S5).

\section{SAFETY}

A total of $77.1 \%$ of the patients in the tezepelumab group and $80.8 \%$ of those in the placebo group reported an adverse event, and $9.8 \%$ and $13.7 \%$ reported a serious adverse event, respectively (Table S6). The percentage of patients who discontinued the trial regimen was $6.8 \%$ in the tezepelumab group and $10.7 \%$ in the placebo group (Table S7). The percentage of patients who discontinued owing to adverse events was $2.1 \%$ in the tezepelumab group and $3.6 \%$ in the placebo group. The most common adverse events were nasopharyngitis, upper respiratory tract infection, headache, and asthma (which was more frequently observed in the placebo group than in the tezepelumab group). All reported serious adverse events are shown in Table S8.

The incidence of severe infections did not differ between the trial groups (46 patients [8.7\%] in each group), nor did the incidence of cancer (4 patients $[0.8 \%]$ in each group). Two deaths were reported during the trial period (one from heart failure and one of unknown cause); both were in the placebo group.

Injection-site reactions occurred in $3.6 \%$ of the patients in the tezepelumab group and in $2.6 \%$ 
Table 2. Change from Baseline to Week 52 in Secondary End Points.:

\begin{tabular}{|c|c|c|}
\hline Variable & $\begin{array}{l}\text { Tezepelumab } \\
(\mathrm{N}=528)\end{array}$ & $\begin{array}{l}\text { Placebo } \\
(\mathrm{N}=531)\end{array}$ \\
\hline \multicolumn{3}{|l|}{ Key secondary end points' } \\
\hline \multicolumn{3}{|l|}{ Prebronchodilator $\mathrm{FEV}_{1}+$} \\
\hline Mean at baseline - liters & $1.8 \pm 0.7$ & $1.9 \pm 0.7$ \\
\hline \multicolumn{3}{|l|}{ Change from baseline at wk 52} \\
\hline No. of patients evaluated & 528 & 531 \\
\hline Least-squares mean — liters & $0.23 \pm 0.02$ & $0.09 \pm 0.02$ \\
\hline Least-squares mean difference vs. placebo $(95 \% \mathrm{Cl})$ - liters & $0.13(0.08$ to 0.18$) \int$ & \\
\hline \multicolumn{3}{|l|}{ ACQ-6 scoreq } \\
\hline Mean at baseline & $2.8 \pm 0.8$ & $2.8 \pm 0.8$ \\
\hline \multicolumn{3}{|l|}{ Change from baseline at wk 52} \\
\hline No. of patients evaluated & 528 & 531 \\
\hline Least-squares mean & $-1.55 \pm 0.05$ & $-1.22 \pm 0.05$ \\
\hline Least-squares mean difference vs. placebo $(95 \% \mathrm{Cl})$ & $-0.33(-0.46$ to -0.20$) \int$ & \\
\hline \multicolumn{3}{|l|}{$\mathrm{AQLQ}(\mathrm{S})+12$ overall score } \\
\hline Mean at baseline & $3.9 \pm 1.0$ & $3.9 \pm 1.0$ \\
\hline \multicolumn{3}{|l|}{ Change from baseline at wk 52} \\
\hline No. of patients evaluated & 527 & 529 \\
\hline Least-squares mean & $1.49 \pm 0.05$ & $1.15 \pm 0.05$ \\
\hline Least-squares mean difference vs. placebo $(95 \% \mathrm{Cl})$ & $0.34(0.20$ to 0.47$) \int$ & \\
\hline \multicolumn{3}{|l|}{ ASD overall score\| } \\
\hline Mean at baseline & $1.4 \pm 0.7$ & $1.4 \pm 0.7$ \\
\hline \multicolumn{3}{|l|}{ Change from baseline at wk 52} \\
\hline No. of patients evaluated & 528 & 531 \\
\hline Least-squares mean & $-0.71 \pm 0.03$ & $-0.59 \pm 0.03$ \\
\hline Least-squares mean difference vs. placebo $(95 \% \mathrm{Cl})$ & $-0.12(-0.19 \text { to }-0.04)^{* k t}$ & \\
\hline \multicolumn{3}{|l|}{ Other secondary end points } \\
\hline \multicolumn{3}{|l|}{ FENO level } \\
\hline Mean at baseline - ppb & $41.4 \pm 36.3$ & $46.3 \pm 44.7$ \\
\hline \multicolumn{3}{|l|}{ Change from baseline at wk 52} \\
\hline No. of patients evaluated & 440 & 426 \\
\hline Least-squares mean - ppb & $-17.3 \pm 1.2$ & $-3.5 \pm 1.2$ \\
\hline Least-squares mean difference vs. placebo $(95 \% \mathrm{Cl})-\mathrm{ppb}$ & $-13.8(-17.1$ to -10.6$)$ & \\
\hline \multicolumn{3}{|l|}{ Blood eosinophil count } \\
\hline Mean at baseline - cells $/ \mu l$ & $327 \pm 293$ & $353 \pm 488$ \\
\hline \multicolumn{3}{|l|}{ Change from baseline at wk 52} \\
\hline No. of patients evaluated & 458 & 451 \\
\hline Least-squares mean - cells $/ \mu \mid$ & $-170 \pm 9$ & $-40 \pm 9$ \\
\hline Least-squares mean difference vs. placebo $(95 \% \mathrm{Cl})-$ cells $/ \mu \mid$ & $-130(-156$ to -104$)$ & \\
\hline \multicolumn{3}{|l|}{ Serum total IgE } \\
\hline Mean at baseline - IU/ml & $515.7 \pm 959.8$ & $614.1 \pm 1159.5$ \\
\hline \multicolumn{3}{|l|}{ Change from baseline at wk 52} \\
\hline No. of patients evaluated & 482 & 471 \\
\hline
\end{tabular}


Table 2. (Continued.)

Variable

Tezepelumab

Placebo

$(\mathrm{N}=528)$

$(\mathrm{N}=531)$

Least-squares mean — IU/ml

$-164.4 \pm 34.4$

$43.6 \pm 34.5$

Least-squares mean difference vs. placebo $(95 \% \mathrm{Cl})-\mathrm{IU} / \mathrm{ml} \quad-208.0(-303.7$ to -112.3$)$

* Plus-minus values are means \pm SD (for values at baseline) or least-squares means \pm SE (for change from baseline at week 52).

$\dagger$ The change from baseline for each of the key secondary end points (prebronchodilator FEV $\mathrm{F}_{1}$ and scores on the ACQ-6, AQLQ[S]+12, and Asthma Symptom Diary [ASD]) was analyzed with the use of a repeated-measures model, after multiple imputation to complete the follow-up under the assumption that data were missing at random. The response variable was the change from baseline at each scheduled visit after randomization up to and including week 52 (irrespective of whether the patient continued to receive the trial regimen or received other treatments). Trial-group assignment, visit, geographic region, age (adolescents or adults), and treatment-by-visit interaction were included as factors, and the baseline of the corresponding end point was included as a continuous linear covariate in the model. Adjusted means were calculated from the model with the use of the observed-margins approach, in which the contribution of model factors to the estimate is weighted proportionally to the presence of these factors in the data. For the ASD score, each of the 52 weeks used for the weekly mean calculation replaced visit in the above model specification.

+ The minimum clinically important difference is 0.1 liters.

$\int P<0.001$.

I The minimum clinically important difference is 0.5 points.

| ASD scores (reflecting daytime severity, daytime frequency, and nighttime severity) range from 0 (no symptoms) to 4 (worst possible symptoms). The ASD score is the mean of the 10 items. Responses for all 10 items are required to calculate the ASD score; otherwise, it is treated as missing. The minimum clinically important difference is 0.5 points. *** $\mathrm{P}=0.002$.

ti Other secondary end points were assessed with the use of a repeated-measures model. Trial-group assignment, visit, geographic region, age (adolescents or adults), and treatment-by-visit interaction were included as factors, and the baseline of the corresponding end point was included as a continuous linear covariate in the model.

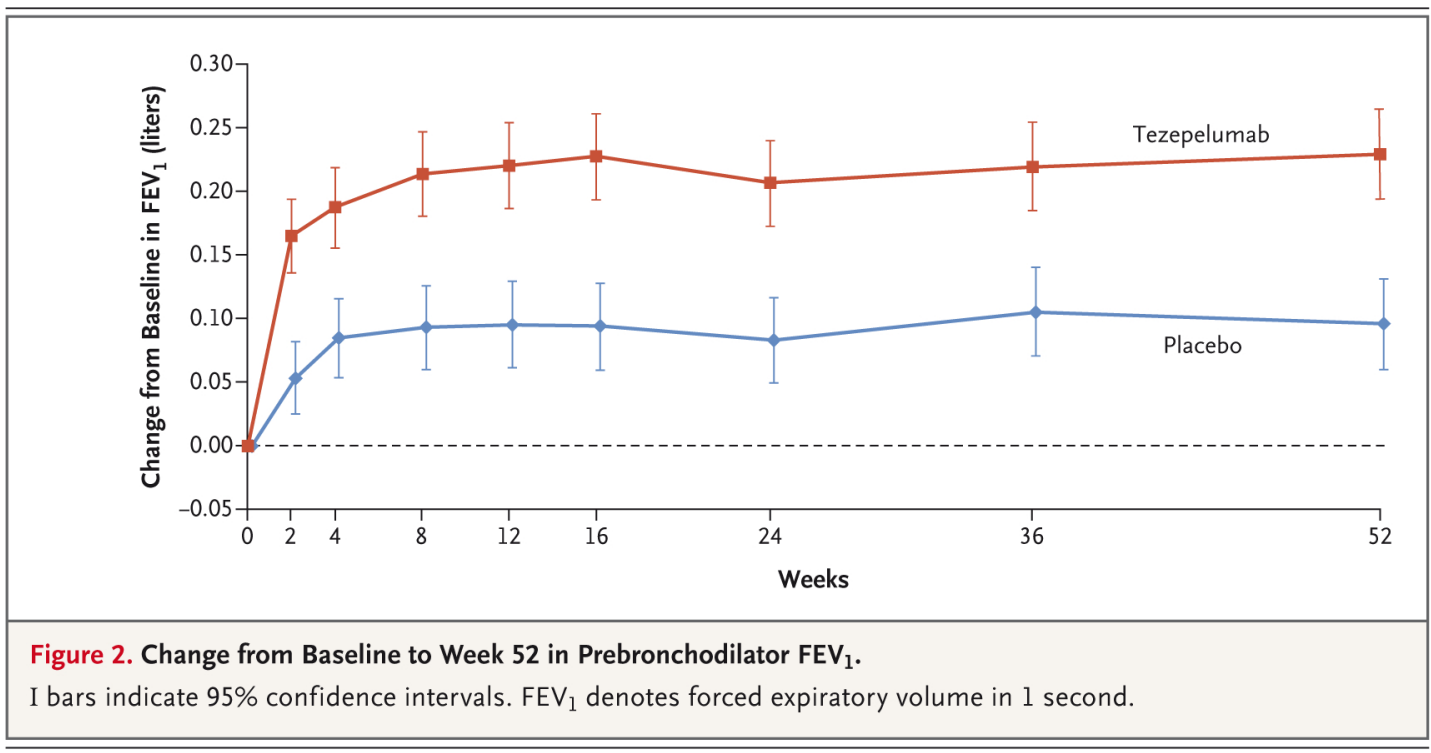

of those in the placebo group (Table S9). No treatment-related anaphylactic reactions or cases of Guillain-Barré syndrome were reported.

At or after baseline, $4.9 \%$ of the patients in the tezepelumab group and $8.3 \%$ of those in the placebo group were positive for antidrug antibodies (Table S10). Neutralizing antibodies were detected in one patient in each group.

\section{DISCUSSION}

The annualized rate of asthma exacerbations was significantly lower with tezepelumab than with placebo among adults and adolescents with severe, uncontrolled asthma, including those with low blood eosinophil counts ( $<300$ cells per microliter) at baseline. Significant improvements 
in $\mathrm{FEV}_{1}$ and scores on the ACQ- $6, \mathrm{AQLQ}(\mathrm{S})+12$, and ASD and substantial reductions in exacerbations that resulted in hospitalization or an emergency department visit were observed with tezepelumab as compared with placebo. The frequencies and types of adverse events did not differ meaningfully between the tezepelumab and placebo groups.

The observed reductions in exacerbations irrespective of baseline blood eosinophil count confirm previous findings from the PATHWAY trial. ${ }^{16}$ Currently available biologics have not been shown to consistently reduce exacerbations in patients with blood eosinophil counts of less than 150 cells per microliter..$^{29-33}$

Tezepelumab simultaneously reduced blood eosinophil count and levels of FeNO and IgE; these findings suggest that the drug suppresses multiple inflammatory pathways. The effect of tezepelumab on these biomarker levels may be related to decreased interleukin-5 and interleukin-13 levels. ${ }^{34}$ The observed reduction in total IgE levels may be due to reduced interleukin-4 and interleukin- 13 levels, causing a progressive reduction in B-cell switching from IgM to IgE isotype production. ${ }^{35}$ These data support the concept that TSLP inhibition may have broader physiological effects than targeting individual T2 cytokines.

The role of TSLP as an upstream communicator between airway structural cells and immune cells in response to stimuli (e.g., allergens, viruses, airborne particles, and trauma) ${ }^{8}$ suggests allergic and nonallergic mechanisms by which tezepelumab may normalize local inflammation, irrespective of blood eosinophil count. Blocking TSLP is expected to markedly reduce T2 cytokine production by $\mathrm{T}$ helper 2 memory cells, innate lymphoid type 2 cells, and mast cells across a spectrum of inflammation. The role of TSLP in mediating $\mathrm{T} 2$ and $\mathrm{T} 17$ responses through dendritic cells, in addition to cross-talk between mast cells and airway smooth muscle cells, are mechanisms potentially relevant to inflammation in populations with low eosinophil counts. ${ }^{8,12,14}$

A strength of our trial is the consistent significant improvements with tezepelumab treatment as compared with placebo across measures of exacerbations, lung function, asthma control, and health-related quality of life. In addition, this was a large, global trial that involved adolescents and adults across the full spectrum of blood eosinophil counts who had severe asthma $(\geq 2$ previous exacerbations). Reductions in the annualized rate of asthma exacerbations were greater in the PATHWAY trial than in the NAVIGATOR trial, potentially owing to the smaller number of patients in the PATHWAY trial. Our trial was limited by its duration (52 weeks), strict inclusion criteria (which excluded relevant patient subgroups - e.g., smokers and those with certain coexisting conditions), and a small number of adolescent patients.

Tezepelumab significantly reduced exacerbations in adults and adolescents with severe, uncontrolled asthma, including those with low blood eosinophil counts, and improved lung function, asthma control, and health-related quality of life.

Supported by AstraZeneca and Amgen.

Dr. Menzies-Gow reports receiving grants, advisory board fees, lecture fees, and consulting fees from and serving as a trial investigator for AstraZeneca, receiving advisory board fees from GlaxoSmithKline, receiving advisory board fees and lecture fees from Novartis, receiving lecture fees from Roche, receiving advisory board fees, lecture fees, and remuneration for travel and accommodation during conference attendance from Teva, receiving advisory board fees and consulting fees from Sanofi, and receiving consulting fees from Vectura; Dr. Corren, receiving grant support, consulting fees, fees for serving on a speakers bureau, and advisory board fees from AstraZeneca and Regeneron, grant support, advisory board fees, and fees for serving on a speakers bureau from Genentech, and grant support from Sanofi, Teva Pharmaceutical Industries, and Optinose; Dr. Bourdin, receiving grant support and consulting fees from and serving as a trial investigator for AstraZeneca, Boehringer Ingelheim, GlaxoSmithKline, Novartis, and SanofiRegeneron, receiving grant support from Cephalon-Teva, receiving consulting fees from and serving as a trial investigator for Actelion, Chiesi, Roche, and Merck, receiving consulting fees from MedinCell, and serving as a trial investigator for Galapagos and Vertex; Dr. Chupp, receiving lecture fees and consulting fees from AstraZeneca, Amgen, Boehringer Ingelheim, Genentech, Genzyme, GlaxoSmithKline, Novartis, Regeneron, Sanofi, and Teva; Dr. Israel, receiving consulting fees from 4D Pharma, Equillium, GlaxoSmithKline, Pneuma Respiratory, Regeneron Pharmaceuticals, and Sienna Biopharmaceuticals, receiving grant support and consulting fees from Novartis, AstraZeneca, and Sanofi, receiving consulting fees and donated drugs from Teva, receiving grant support from Genentech, and owning stock options in Vorso; Dr. Wechsler, receiving consulting fees from AstraZeneca, Boehringer Ingelheim, Cohero Health, Equillium, Genentech, GlaxoSmithKline, Novartis, Regeneron, Sanofi-Genzyme, Sentien Biotechnologies, Teva, and Amgen; Dr. Brightling, receiving grant support and consulting fees from AstraZeneca; Dr. Griffiths, Ms. Hellqvist, and Ms. Bowen, being employed by AstraZeneca; Dr. Kaur, being employed by Amgen; and Ms. Almqvist and Drs. Ponnarambil and Colice, being employed by AstraZeneca. No other potential conflict of interest relevant to this article was reported.

Disclosure forms provided by the authors are available with the full text of this article at NEJM.org.

A data sharing statement provided by the authors is available with the full text of this article at NEJM.org.

We thank the trial patients and their families; the investigators and site staff; Gillian Hunter, Gennaro Ruberto, Joanne Burke, and Yun Chon for their contributions to the trial design and statistical analyses; and Madeleine Wynn of PharmaGenesis London for medical writing support with an earlier version of the manuscript. 


\section{REFERENCES}

1. Global Initiative for Asthma. 2020 GINA main report: global strategy for asthma management and prevention. 2020 (http://ginasthma.org/gina-reports/).

2. Chen S, Golam S, Myers J, Bly C, Smolen $\mathrm{H}, \mathrm{Xu}$ X. Systematic literature review of the clinical, humanistic, and economic burden associated with asthma uncontrolled by GINA Steps 4 or 5 treatment. Curr Med Res Opin 2018;34:2075-88.

3. Carr TF, Zeki AA, Kraft M. Eosinophilic and noneosinophilic asthma. Am J Respir Crit Care Med 2018;197:22-37.

4. Hinks TSC, Levine SJ, Brusselle GG. Treatment options in type-2 low asthma. Eur Respir J 2021;57(1):2000528.

5. Allakhverdi $Z$, Comeau MR, Jessup HK, et al. Thymic stromal lymphopoietin is released by human epithelial cells in response to microbes, trauma, or inflammation and potently activates mast cells. J Exp Med 2007;204:253-8.

6. Soumelis V, Reche PA, Kanzler H, et al. Human epithelial cells trigger dendritic cell mediated allergic inflammation by producing TSLP. Nat Immunol 2002;3: 673-80.

7. Shikotra A, Choy DF, Ohri CM, et al. Increased expression of immunoreactive thymic stromal lymphopoietin in patients with severe asthma. J Allergy Clin Immunol 2012;129(1):104.e1-111.e9.

8. Gauvreau GM, Sehmi R, Ambrose CS, Griffiths JM. Thymic stromal lymphopoietin: its role and potential as a therapeutic target in asthma. Expert Opin Ther Targets 2020;24:777-92.

9. Liu S, Verma M, Michalec L, et al. Steroid resistance of airway type 2 innate lymphoid cells from patients with severe asthma: the role of thymic stromal lymphopoietin. J Allergy Clin Immunol 2018; 141(1):257.e6-268.e6.

10. Kitajima M, Lee HC, Nakayama T, Ziegler SF. TSLP enhances the function of helper type 2 cells. Eur J Immunol 2011; 41:1862-71.

11. Liang Y, Yu B, Chen J, et al. Thymic stromal lymphopoietin epigenetically upregulates Fc receptor $\gamma$ subunit-related receptors on antigen-presenting cells and induces $\mathrm{T}_{\mathrm{H}} 2 / \mathrm{T}_{\mathrm{H}} 17$ polarization through dectin-2. J Allergy Clin Immunol 2019; 144(4):1025.e7-1035.e7.

12. Tanaka J, Watanabe N, Kido M, et al. Human TSLP and TLR3 ligands promote differentiation of Th17 cells with a central memory phenotype under Th2-polarizing conditions. Clin Exp Allergy 2009; 39:89-100.

13. West EE, Spolski R, Kazemian M, Yu ZX, Kemper C, Leonard WJ. A TSLP-com- plement axis mediates neutrophil killing of methicillin-resistant Staphylococcus aureus. Sci Immunol 2016;1(5):eaaf8471.

14. Allakhverdi $Z$, Comeau MR, Jessup HK, Delespesse G. Thymic stromal lymphopoietin as a mediator of crosstalk between bronchial smooth muscles and mast cells. J Allergy Clin Immunol 2009; 123(4):958.e2-960.e2.

15. Gauvreau GM, O'Byrne PM, Boulet L-P, et al. Effects of an anti-TSLP antibody on allergen-induced asthmatic responses. N Engl J Med 2014;370:2102-10.

16. Corren J, Parnes JR, Wang L, et al. Tezepelumab in adults with uncontrolled asthma. N Engl J Med 2017;377:936-46.

17. Corren J, Garcia Gil E, Parnes JR, Pham T-H, Griffiths JM. Tezepelumab treatment effect on annualized rate of exacerbations by baseline biomarkers in uncontrolled severe asthma patients: phase 2b PATHWAY study. Am J Respir Crit Care Med 2019;199:A2621. abstract.

18. Corren J, Liu MC, Bowen K, Sałapa K, Colice G, Llanos-Ackert JP. Efficacy of tezepelumab in patients with low and high bronchodilator reversibility in PATHWAY. Eur Respir J 2020;56:Suppl 64:2269. abstract.

19. Corren J, Ambrose C, Sałapa K, Parnes J, Colice G. Tezepelumab efficacy in severe asthma by specific allergen sensitivity: results from the PATHWAY study. Ann Allergy Asthma Immunol 2020;125:Suppl: S5-S6. abstract.

20. Menzies-Gow A, Colice G, Griffiths JM, et al. NAVIGATOR: a phase 3 multicentre, randomized, double-blind, placebocontrolled, parallel-group trial to evaluate the efficacy and safety of tezepelumab in adults and adolescents with severe, uncontrolled asthma. Respir Res 2020;21:266. 21. Ly N, Zheng Y, Griffiths JM, et al. Pharmacokinetic and pharmacodynamic modeling of tezepelumab to guide phase 3 dose selection for patients with severe asthma. J Clin Pharmacol 2020 December 28 (Epub ahead of print).

22. Tepper RS, Wise RS, Covar R, et al. Asthma outcomes: pulmonary physiology. J Allergy Clin Immunol 2012;129:Suppl: S65-S87.

23. American Thoracic Society. Asthma Control Questionnaire (http://www.thoracic .org/members/assemblies/assemblies/srn/ questionaires/acq.php).

24. Juniper EF, Buist AS, Cox FM, Ferrie PJ, King DR. Validation of a standardized version of the Asthma Quality of Life Questionnaire. Chest 1999;115:1265-70.

25. Globe G, Martin M, Schatz M, et al. Symptoms and markers of symptom se- verity in asthma - content validity of the asthma symptom diary. Health Qual Life Outcomes 2015;13:21.

26. Globe G, Wiklund I, Lin J, et al. Psychometric properties of the Asthma Symptom Diary (ASD), a diary for use in clinical trials of persistent asthma. J Allergy Clin Immunol Pract 2016;4(1):60. e4-66.e4.

27. Globe G, Wiklund I, Mattera M, Zhang H, Revicki DA. Evaluating minimal important differences and responder definitions for the Asthma Symptom Diary in patients with moderate to severe asthma. J Patient Rep Outcomes 2019;3:22.

28. Cleveland WS. Robust locally weighted regression and smoothing scatterplots. J Am Stat Assoc 1979;74:829-36.

29. Castro M, Corren J, Pavord ID, et al. Dupilumab efficacy and safety in moderate-to-severe uncontrolled asthma. N Engl J Med 2018;378:2486-96.

30. ClinicalTrials.gov. Evaluation of dupilumab in patients with persistent asthma (Liberty Asthma Quest) (https:// clinicaltrials.gov/ct2/show/study/

NCT02414854).

31. Bleecker ER, FitzGerald JM, Chanez P, et al. Efficacy and safety of benralizumab for patients with severe asthma uncontrolled with high-dosage inhaled corticosteroids and long-acting $\beta_{2}$-agonists (SIROCCO): a randomised, multicentre, placebo-controlled phase 3 trial. Lancet 2016;388:2115-27.

32. FitzGerald JM, Bleecker ER, Nair P, et al. Benralizumab, an anti-interleukin-5 receptor $\alpha$ monoclonal antibody, as add-on treatment for patients with severe, uncontrolled, eosinophilic asthma (CALIMA): a randomised, double-blind, placebo-controlled phase 3 trial. Lancet 2016;388: 2128-41.

33. Ortega HG, Yancey SW, Mayer B, et al. Severe eosinophilic asthma treated with mepolizumab stratified by baseline eosinophil thresholds: a secondary analysis of the DREAM and MENSA studies. Lancet Respir Med 2016;4:549-56.

34. Pham T-H, Ren P, Parnes JR, Griffiths JM. Tezepelumab reduces multiple key inflammatory biomarkers in patients with severe, uncontrolled asthma in the phase 2b PATHWAY study. Am J Respir Crit Care Med 2019;199:A2677. abstract.

35. The production of IgE. In: Janeway CA Jr, Travers P, Walport M, Shlomchik MJ. Immunobiology: the immune system in health and disease. 5th ed. New York: Garland Science, 2001 (https://www.ncbi .nlm.nih.gov/books/NBK27117/).

Copyright (c) 2021 Massachusetts Medical Society. 